\title{
Maternal Protein Restriction Increases Hepatic Glycogen Storage in Young Rats
}

\author{
ALISON K. GOSBY, CHRISTOPHER A. MALONEY, JENNY L. PHUYAL, GARETH S. DENYER, \\ JANET M. BRYSON, AND IAN D. CATERSON \\ Human Nutrition Unit, School of Molecular and Microbial Biosciences, The University of Sydney, Sydney, \\ New South Wales 2006, Australia
}

\begin{abstract}
This study aimed to determine whether maternal protein restriction alters hepatic glycogen metabolism. Mated female rats were fed diets containing $20 \%$ protein throughout pregnancy and lactation (CONT), $8 \%$ protein throughout pregnancy and lactation (LP), or $8 \%$ protein during the last week of pregnancy only and lactation (LLP). Weights and lengths were reduced in the LLP and LP offspring compared with the CONT offspring. The LLP and LP offspring demonstrated reduced insulin concentrations at both 10 and $26 \mathrm{~d}$ and also failed to show the increase in insulin seen with time in the CONT offspring. Serum glucose and leptin levels increased with time but were not different among the groups; however, in relation to adiposity leptin levels were greater in the LLP and LP offspring at $26 \mathrm{~d}$. The LLP and LP offspring had increased hepatic glycogen at day 10 (CONT, 75.1 \pm 9.8; LLP, $103.4 \pm 11.0 ; \mathrm{LP}, 116.0 \pm 18.4$ glucose residues $/ \mathrm{g}$ tissue) and d 26 (CONT, $183.1 \pm 38.9$; LLP, $395.3 \pm 16.8$; LP, $396.6 \pm 15.1$ glucose residues/g tissue). Glycogen synthase expression was increased in the LLP and LP offspring at $10 \mathrm{~d}$ but
\end{abstract}

\section{ABSTRACT}

not $26 \mathrm{~d}$; glucose transporter 2 and glycogen phosphorylase expressions were not different at either time. At 26 d glycogen synthase activity was not different; however, glycogen phosphorylase $a$ activity was reduced. The enhanced capacity to store glycogen despite reductions in insulin secretion suggests increased insulin sensitivity possibly acting with an alternative non-insulin-dependent glycogen storage mechanism. (Pediatr Res 54: 413-418, 2003)

\section{CONT, control}

\section{Abbreviations}

LLP, late low protein

LP, low protein

GS, glycogen synthase

GP, glycogen phosphorylase

GLUT2, glucose transporter 2

$\mathbf{P R}$, protein restriction
There is now considerable evidence from animal models to support a role for maternal undernutrition in fetal programming such that alterations in metabolism during gestation or lactation result in metabolic changes in the offspring in adult life $(1,2)$. The thrifty phenotype hypothesis postulates that these changes occur to ensure a sufficient supply of energy to the brain and other vital organs; these changes may be beneficial in times of starvation, but detrimental in times of overnutrition in later life (3).

One commonly used animal model of maternal undernutrition is the isocaloric PR rat (4). This model involves feeding dams an isocaloric diet containing only $40 \%$ of the protein of the control diet. Offspring of PR dams have been shown to develop insulin resistance as adults, with this insulin resistance

Received November 15, 2002; accepted March 5, 2003

Correspondence: Alison Gosby, M.D., Human Nutrition Unit, School of Molecular and Microbial Biosciences, University of Sydney, Sydney NSW 2006, Australia; e-mail: agos7353@mail.usyd.edu.au

Supported by a National Health and Medical Research Council of Australia grant.

DOI: 10.1203/01.PDR.0000077470.63060.9B being preceded by a period of improved insulin sensitivity and greater glucose tolerance $(5,6)$. As well as changes in whole body glucose metabolism, tissue-specific changes in gene expression and the activities of enzymes involved in glucose homeostasis have also been reported $(5,7)$.

The timing of an insult such as PR may also be of importance. Crossover studies have suggested that the effects of protein deprivation in utero may differ from the effects of protein deprivation during lactation (7). It may also be that the final trimester of pregnancy is the crucial time of deprivation. The Dutch famine study has shown that exposure to famine throughout mid to late gestation results in reduced insulin sensitivity and glucose tolerance in comparison with exposure throughout early gestation (8).

Most previous studies have examined the effects of maternal undernutrition on offspring in adult life $(5,7,9)$, but few studies have looked to see what effect maternal PR has had on glucose metabolism during neonatal life and at the time of weaning (7). The first objective of this study was to determine whether maternal PR results in favored hepatic glycogen stor- 
age in suckling pups after exposure to PR throughout late gestation and lactation or the whole of gestation and lactation. The second objective was to determine whether this change occurs via changes in expression and activity of insulinsensitive genes involved in glycogen metabolism.

\section{METHODS}

Diets. Compositions of the diets used in this study are detailed in Table 1 . The CONT diet contained $21 \%$ of calories as protein whereas in the LP diet, only $8.3 \%$ of energy was in the form of protein. The diets were isocaloric with the difference in energy content being made up with carbohydrate. The diets were fed ad libitum in powdered form.

Experimental animals. Approval for this study was obtained from the University of Sydney animal ethics committee. All animals were housed in a 12-h/12-h light-dark cycle room maintained at $22^{\circ} \mathrm{C}$. Mated female Wistar rats were placed on either the CONT or LP diet at conception and remained on this diet for the whole of the gestational and lactation period. A third group received the CONT diet for the first 2 wk of pregnancy but was then switched to the LP diet for the last week of pregnancy and during lactation (LLP group). Any litters smaller than eight or larger than 15 were not included in the study. The study included five CONT, eight LLP, and eight LP litters. The average litter size for the study was $12.6 \pm 0.4$, and there was no difference in litter sizes among the diet groups $(\mathrm{CONT}, 13.6 \pm 0.6$; LLP, $11.6 \pm 0.5$; and LP, $12.7 \pm 0.9)$. Body weights and lengths of all offspring were measured once a week until weaning. At 10 and $26 \mathrm{~d}$, one to two male offspring were taken from each litter after free access to food and killed by Nembutal in an anesthesia dose. After removal of 10-d pups the average litter size was $11.1 \pm 0.4$ with no difference in litter size among the diet groups (CONT, $11.8 \pm$ 0.7; LLP, $10.4 \pm 0.6$; and LP, $11.5 \pm 0.9$ ). Blood was collected by cardiac puncture, and serum was separated and stored at $-20^{\circ} \mathrm{C}$ for subsequent measurement of hormone and metabolite levels. Epididymal, peritoneal, and subcutaneous fat pad weights were measured at $26 \mathrm{~d}$; weights were not measured at $10 \mathrm{~d}$ owing to insufficient size. The liver was weighed and a portion was collected, immediately freeze-clamped, and stored at $-80^{\circ} \mathrm{C}$ for subsequent assay of gene expression and enzyme activities at $26 \mathrm{~d}$, and gene expression only at $10 \mathrm{~d}$ owing to insufficient liver size.

Table 1. Composition of CONT and LP diets fed to mothers during pregnancy and lactation

\begin{tabular}{lcc}
\hline & CONT & LP \\
\cline { 2 - 3 } & $\mathrm{g} / 100 \mathrm{~g}$ & $\mathrm{~g} / 100 \mathrm{~g}$ \\
\hline Casein & 22 & 9 \\
D L Methionine & 0.3 & 0.08 \\
Cerelose & 55.7 & 68.9 \\
Cornstarch & 8 & 8 \\
Soybean oil & 4.3 & 4.3 \\
Cellulose & 5 & 5 \\
Minerals & 3.5 & 3.5 \\
Vitamins & 1 & 1 \\
Choline bitartrate & 0.2 & 0.2 \\
\hline
\end{tabular}

Serum measurements. Glucose was measured using the glucose oxidase method. Both insulin and leptin were measured by RIA (Linco Research, St. Louis, MO, U.S.A.).

Liver glycogen content. Liver glycogen content was measured using the filter paper method of Chan and Exton (10).

Measurement of gene expression. Expression of mRNA of the genes of interest was determined in liver tissue using semiquantitative reverse transcription PCR techniques as previously described (11). The genes of interest were chosen because of their role in 1) glucose uptake (GLUT2), 2) glycogen synthesis (GS), and 3) glycogen breakdown (GP). Primer sequences for GS and GP were designed specifically for this study, with a previously published sequence being used for GLUT2 (12). Primer sequences and cycles numbers for each gene are shown in Table 2. Products were analyzed after agarose gel electrophoresis and staining with ethidium bromide. Band densities of PCR products were measured using Quantity 1 quantitation software (BioRad Laboratories, Hercules, CA, U.S.A.). Results are expressed as the ratio of the intensity of the band of the gene of interest to the intensity of the $\beta$-actin band.

Measurement of enzyme activities. Hepatic GS activity was measured radiochemically by determining the rate of incorporation of uridine diphosphate glucose into glycogen in the presence of $0.01 \mathrm{mM}$ (active, GSa) and $10 \mathrm{mM}$ (total, GSt) glucose-6-phosphate (13). The fractional velocity of GS was also determined (GSa/GSt). Hepatic GPa activity was determined in the reverse direction by measuring the incorporation of glucose-1-phosphate into glycogen (14).

Data analyses. All data are expressed as mean \pm SEM. To test for differences among diet groups results were analyzed by one-way ANOVA using Fisher's protected least-significance differences post hoc test to test for differences between individual groups. The $t$ test was used to test for age differences among offspring on the same diet. Differences with a $p$ value less than 0.05 were considered significant.

\section{RESULTS}

Effect of maternal PR on body weight and length. Weights and lengths were measured at birth and wk 1, 2, and 3 on all offspring (Fig. 1). Birth weights (in grams) (CONT, $6.7 \pm 0.1$; LLP, $6.2 \pm 0.1$; LP, $5.8 \pm 0.1 ; p<0.01 \mathrm{LLP}$ and LP versus CONT) and lengths in cm (CONT, $7.0 \pm 0.1$; LLP, $6.8 \pm 0.1$; $\mathrm{LP}, 6.6 \pm 0.1 ; p<0.001 \mathrm{LLP}$ and LP versus CONT) were

Table 2. PCR primer sequences and cycle times required to ensure linearity of amplification for liver in reverse transcription PCR for the measurement of $m R N A$ expression in liver

\begin{tabular}{|c|c|c|}
\hline Gene & Sequence & $\begin{array}{l}\text { No. of } \\
\text { cycles }\end{array}$ \\
\hline \multirow[t]{2}{*}{ GS } & 5'-CCT CAG TGT GGA ACC TGG AC-3' (forward) & 34 \\
\hline & 5'-CAG TGT GGC ATG GGT TGT AA-3' (reverse) & \\
\hline \multirow[t]{2}{*}{ GP } & 5'-GAT GGT GTA GGA ACC GTG TT-3' (forward) & 34 \\
\hline & 5'-ATG CGG TCG ATG TCT TTA GG-3' (reverse) & \\
\hline \multirow[t]{2}{*}{ GLUT2 } & 5'-CAT TGC TGG AAG AAG CGT ATC AG-3' (forward) & 36 \\
\hline & 5'-GAG ACC TTC TGC TCA GTC GAC G-3' (reverse) & \\
\hline \multirow[t]{2}{*}{$\beta$-actin } & 5'-AAT CCT GTG GCA TCC ATG AAA-3' (forward) & 29 \\
\hline & 5'-CGC AGC TCA GTA ACA GTC GC-3' (reverse) & \\
\hline
\end{tabular}



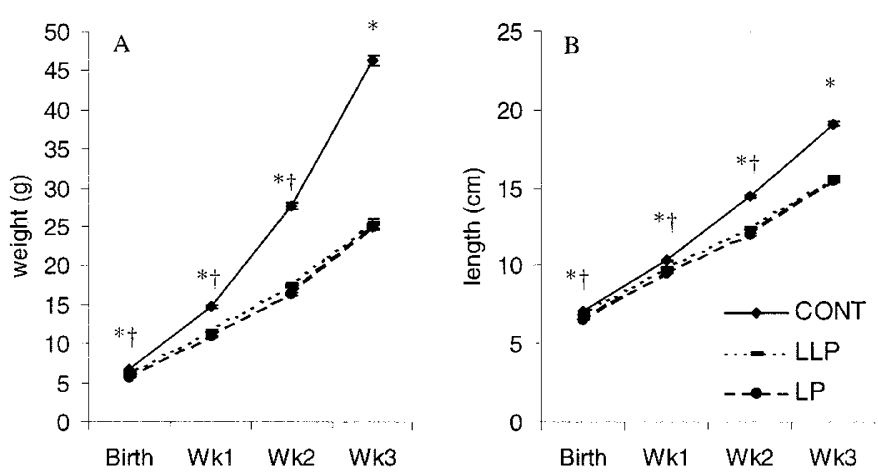

Figure 1. Body weights $(A)$ and lengths $(B)$ at birth and throughout lactation of offspring of dams fed $20 \%$ protein (CONT, $n=82$ ), $8 \%$ protein throughout pregnancy and lactation $(L P, n=76)$, or $8 \%$ protein during the last week of pregnancy and lactation ( $L L P, n=93) .{ }^{*} p<0.0005$, LLP and LP $v s$ CONT. $\dagger p<0.05$ LLP $v$ s LP.

significantly reduced in the LLP and LP groups compared with the CONT offspring. This reduction in weight and length in the LLP and LP groups was also seen at wk 1, 2, and 3. The weights and lengths of the LP offspring were also significantly reduced when compared with the LLP offspring at birth, wk 1, and wk 2.

Effect of maternal PR on liver and white adipose tissue weights. Liver weights were significantly reduced in the LLP and LP groups at 10 and $26 \mathrm{~d}$ compared with CONT offspring; however, when expressed in relation to total body weight the difference was no longer present (Table 3). Epididymal, peritoneal, subcutaneous, and total fat pads were also significantly reduced in the 26-d LLP and LP offspring; in relation to body weight, the difference was only maintained in the LP offspring for epididymal and peritoneal fat pads.

Effect of maternal PR on serum metabolite and hormone levels in 10-d and 26-d offspring. There was no effect of maternal PR on serum glucose levels at either time nor was

Table 3. Effect of maternal PR on liver, epididymal, peritoneal, subcutaneous, and total white adipose tissue weights

\begin{tabular}{|c|c|c|c|}
\hline & CONT & LLP & LP \\
\hline \multicolumn{4}{|l|}{$10-\mathrm{d}$} \\
\hline (n) & (8) & (12) & (10) \\
\hline Liver (mg) & $647.6 \pm 28.4$ & $408.8 \pm 23.5 \dagger$ & $427.4 \pm 67.1 \dagger$ \\
\hline Liver:BW (mg/g) & $30.4 \pm 0.7$ & $27.4 \pm 0.9$ & $30.8 \pm 4.6$ \\
\hline \multicolumn{4}{|l|}{$26-d$} \\
\hline (n) & (9) & (11) & (10) \\
\hline Liver (mg) & $3222 \pm 128.4$ & $1565 \pm 95.9^{*}$ & $1425 \pm 72.8^{*}$ \\
\hline Liver:BW (mg/g) & $46.2 \pm 1.9$ & $44.5 \pm 2.1$ & $41.7 \pm 0.7$ \\
\hline Total WAT (mg) & $1378 \pm 170.0$ & $722.0 \pm 87.9^{*}$ & $579.9 \pm 57.6^{*}$ \\
\hline Total WAT:BW (mg/g) & $19.3 \pm 2.0$ & $18.8 \pm 1.6$ & $16.7 \pm 1.0$ \\
\hline Epididymal (mg) & $221.0 \pm 30.7$ & $85.0 \pm 10.2^{*}$ & $57.5 \pm 7.0^{*}$ \\
\hline Epididymal:BW (mg/g) & $3.1 \pm 0.4$ & $2.3 \pm 0.3$ & $1.7 \pm 0.1 \dagger$ \\
\hline Peritoneal $(\mathrm{mg})$ & $197.6 \pm 20.8$ & $90.7 \pm 10.5^{*}$ & $72.4 \pm 8.1^{*}$ \\
\hline Peritoneal:BW (mg/g) & $2.8 \pm 0.3$ & $2.4 \pm 0.2$ & $2.1 \pm 0.2 \ddagger$ \\
\hline Subcutaneous (mg) & $960.1 \pm 124.0$ & $508.6 \pm 62.5^{*}$ & $450.0 \pm 45.3 *$ \\
\hline Sub:BW (mg/g) & $13.4 \pm 1.5$ & $13.6 \pm 1.1$ & $13.0 \pm 0.8$ \\
\hline
\end{tabular}

Data are mean \pm SEM. $* p<0.0005, \dagger p<0.005, \$ p<0.02$ LLP and LP offspring vs CONT offspring of same age.

Abbreviations used: BW, body weight; Sub, subcutaneous; WAT, white adipose tissue. there any difference between $\mathrm{d} 10$ and d 26 in any group (Table 4). Serum insulin levels were reduced by approximately $50 \%$ in LLP and LP groups at $\mathrm{d} 10$. Whereas insulin levels in CONT had doubled by $\mathrm{d} 26$, there was no increase in insulin levels in either LLP or LP offspring, such that by d 26 levels in LLP and LP offspring were only $25 \%$ of those in CONT offspring. Serum leptin levels increased approximately 2 -fold between $\mathrm{d}$ 10 and 26 in all three groups with no effect of maternal PR at either time. However, LLP and LP offspring had higher leptin levels at $26 \mathrm{~d}$ after correction for adiposity (total fat pad weight).

Effect of maternal PR on hepatic glycogen content. At both 10 and $26 \mathrm{~d}$, LLP and LP offspring were found to have increased liver glycogen content compared with the CONT offspring (Fig. 2). There was a 2.5 -fold increase in glycogen content in CONT offspring between d 10 and 26, with an even greater increase in both LLP and LP groups. When adjusted for the size of the liver, total liver glycogen (total liver glucosyl residues) was not different among the groups at either 10 (LLP, $43.4 \pm 6.3$; LP, $42.7 \pm 6.9$; CONT, $49.3 \pm 7.0$ ) or $26 \mathrm{~d}$ (LLP, $636.4 \pm 61.3 ; \mathrm{LP}, 564.2 \pm 42.8$; CONT, $629.7 \pm 150.1$ ).

Effect of maternal PR on liver gene expression in 10-d and 26-d pups. GLUT2 expression doubled in all three groups between $\mathrm{d} 10$ and d 26, but there were no differences as a result of maternal PR (Table 5). GS expression did not change with time, but there was an increase in expression in both LLP and LP groups relative to CONT at d 10. GP expression also doubled in all three groups between d 10 and d 26, with no effect of maternal PR at either time.

Effect of maternal PR on the activities of hepatic GS and GP activities in 26-d weanlings. There were no differences in GSa or GSt activity at $26 \mathrm{~d}$ (Table 6). We also found no changes in the fractional velocity of GS. GP $a$ activity was reduced by maternal $\mathrm{PR}$, but this was only significant in the LP group. There was no relationship between expression and enzyme activity for either GS or GPa (data not shown).

\section{DISCUSSION}

The present study confirms that maternal PR results in growth retardation of the offspring. The LLP and LP offspring presented similar reductions in birth weights and lengths, demonstrating the importance of nutrition throughout the third week of gestation. The prominent result in this study was the capacity of LLP and LP offspring to store equivalent amounts of hepatic glycogen despite reduced liver weights and plasma insulin concentrations. This increased capacity to store glycogen in the presence of reduced insulin suggests increased insulin sensitivity; however, the data do not show any changes in expression or in vitro activity of insulin-sensitive enzymes supporting this change. There was a slight reduction in GP $a$ activity in the LP group, although this reduction was not seen in the LLP offspring, suggesting that this was not the major cause of the increased capacity to store glycogen seen in both groups.

The lower insulin levels seen in the LLP and LP offspring are consistent with the reduced pancreatic insulin secretion previously reported in this model (15). In this study, not only 
Table 4. Effect of maternal PR on serum metabolite and hormone levels

\begin{tabular}{|c|c|c|c|}
\hline & CONT & LLP & LP \\
\hline \multicolumn{4}{|c|}{ Glucose $(\mathrm{mM})$} \\
\hline $10 \mathrm{~d}$ & $9.82 \pm 0.43(8)$ & $9.05 \pm 0.23(12)$ & $9.26 \pm 0.37(9)$ \\
\hline $26 \mathrm{~d}$ & $11.25 \pm 0.28(9)$ & $10.64 \pm 0.35(14)$ & $11.04 \pm 0.44(11)$ \\
\hline $10 \mathrm{~d}$ & $568.5 \pm 82.5(8)$ & $106.5 \pm 18.0(9) \dagger$ & $135.0 \pm 18.0(10) \ddagger$ \\
\hline $26 \mathrm{~d}$ & $625.5 \pm 67.5(8)$ & $159.0 \pm 31.5(11)^{*}$ & $144.0 \pm 15.0(10)^{*}$ \\
\hline \multicolumn{4}{|c|}{ Leptin (ng/mL) } \\
\hline \multicolumn{4}{|c|}{ Leptin/fat (ng/mL/mg) } \\
\hline $26 \mathrm{~d}$ & $2.26 \pm 0.28(8)$ & $3.97 \pm 0.46(7)$ & $6.28 \pm 1.11(10) \dagger$ \\
\hline
\end{tabular}

Data are mean $\pm \operatorname{SEM}(n){ }^{*} p<0.0001, \dagger p<0.002, \ddagger p<0.05$, LLP and LP $v s$ CONT offspring of same age. $\S p<0.0001, p<0.005, \uparrow p<0.02,26-\mathrm{d}$ vs 10-d offspring on same diet.

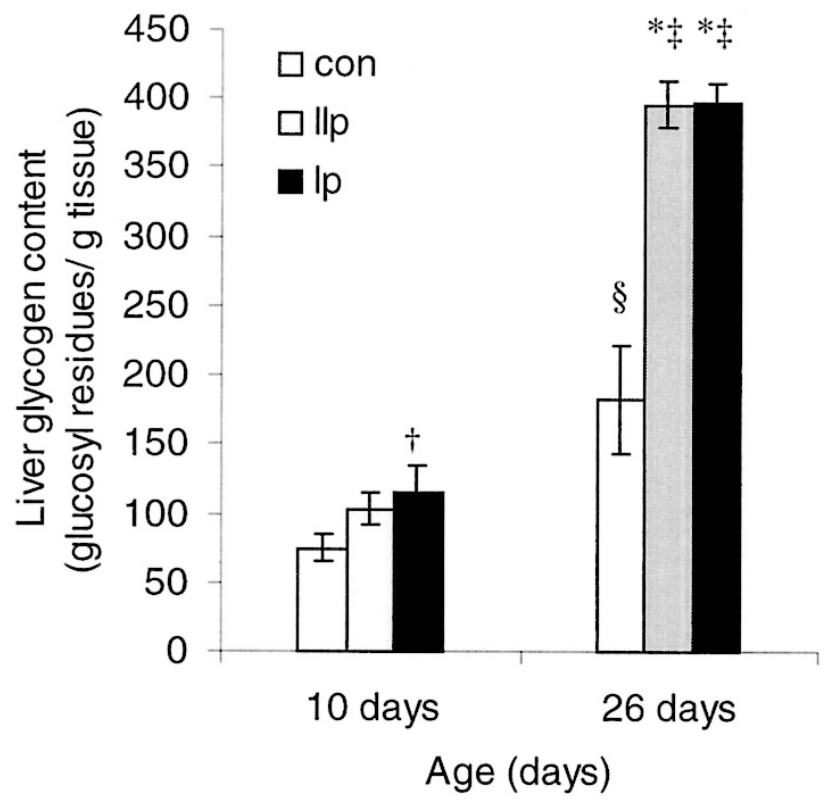

Figure 2. Hepatic glycogen content in 10-d and 26-d offspring of mothers fed $20 \%$ protein $(C O N T, 10-\mathrm{d} n=8,26-\mathrm{d} n=6), 8 \%$ protein throughout pregnancy and lactation $(L P, 10-\mathrm{d} n=8,26-\mathrm{d} n=10)$, or $8 \%$ protein during the last week of pregnancy and lactation $(L L P, 10-\mathrm{d} n=11,26-\mathrm{d} n=10)$. Data represent mean \pm SEM. ${ }^{*} p<0.0001$ and $\dagger p<0.05$ LLP and LP $v s$ CONT offspring at same age. $₫ p<0.0001, \S p<0.0126$-d $v s 10$-d offspring on same diet.

did the LLP and LP offspring have reduced plasma insulin concentrations at both times, they also failed to show the increase in insulin throughout lactation seen in the CONT group. Despite no increase in insulin levels between $\mathrm{d} 10$ and d 26 there was still a 4-fold increase in hepatic glycogen content in the LLP and LP offspring. Also, despite the reduced plasma insulin concentrations in the LLP and LP offspring in this study, there were no changes in plasma glucose concentrations, indicating that the LLP and LP offspring have in all likelihood adapted to their reduced insulin secretion by increasing insulin sensitivity. Glucose tolerance studies in humans and animals have demonstrated increased or equivalent insulin sensitivity and glucose tolerance in young LLP and LP offspring when compared with control offspring $(5,6)$. In vitro
Table 5. Effect of maternal PR on expression of hepatic GS, GP, and GLUT 2

\begin{tabular}{cccc}
\hline & CONT & LLP & LP \\
\hline $10 \mathrm{~d}$ & & $(11)$ & $(5)$ \\
$(\mathrm{n})$ & & $0.68 \pm 0.08$ & $0.68 \pm 0.09$ \\
GLUT2 & $0.86 \pm 0.12$ & $1.11 \pm 0.08^{*}$ & $1.23 \pm 0.06^{*}$ \\
GS & $0.84 \pm 0.10$ & $0.45 \pm 0.04$ & $0.48 \pm 0.04$ \\
GP & $0.47 \pm 0.02$ & $(8)$ & $(9)$ \\
$26 \mathrm{~d}$ & $(5)$ & $1.54 \pm 0.06 \dagger$ & $1.55 \pm 0.12 \dagger$ \\
(n) & $1.47 \pm 0.09 \dagger$ & $1.11 \pm 0.05$ & $1.00 \pm 0.09$ \\
GLUT2 & $0.94 \pm 0.11$ & $0.92 \pm 0.06 \dagger$ & $0.90 \pm 0.06 \dagger$ \\
GS & $0.85 \pm 0.09 \dagger$ & & \\
GP & & &
\end{tabular}

Ratio of specific gene to $\beta$-actin expressed as mean \pm SEM. $* p<0.05$, LLP and LP $v s$ CONT offspring of same age. $\dagger p<0.0005, \ddagger p<0.01,26-\mathrm{d}$ vs 10-d offspring on same diet.

Table 6. Effect of maternal PR on hepatic GS and GP activities

\begin{tabular}{|c|c|c|c|}
\hline & CONT & LLP & LP \\
\hline \multicolumn{4}{|c|}{ GS activity $\left(\mathrm{nmol} \mathrm{UDPG} \cdot \mathrm{min}^{-1} \cdot \mathrm{mg}\right.$ wet $\left.\mathrm{wt}^{-1}\right)$} \\
\hline (n) & (5) & (9) & (9) \\
\hline GSa & $0.77 \pm 0.15$ & $0.74 \pm 0.09$ & $0.73 \pm 0.10$ \\
\hline GSt & $1.29 \pm 0.24$ & $1.32 \pm 0.12$ & $1.11 \pm 0.10$ \\
\hline FV & $0.60 \pm 0.02$ & $0.56 \pm 0.03$ & $0.64 \pm 0.06$ \\
\hline \multicolumn{4}{|c|}{ GP activity $\left(\mathrm{nmol} \mathrm{G1P} \cdot \mathrm{min}^{-1} \cdot \mathrm{mg}\right.$ wet $\left.\mathrm{wt}^{-1}\right)$} \\
\hline (n) & (8) & (14) & $(11)$ \\
\hline $\mathrm{GP} a$ & $12.45 \pm 0.98$ & $10.98 \pm 0.83$ & $8.66 \pm 0.99 *$ \\
\hline
\end{tabular}

Data are mean \pm SEM. $* p<0.05$ LLP and LP $v s$ CONT offspring.

Abbreviations used: FV, fractional velocity of GS; G1P, glucose-1phosphate; UDPG, uridine diphosphate glucose.

perfusion studies of the hepatocyte have shown that at $3 \mathrm{mo}$, LLP and LP offspring display reduced glucagon and increased insulin receptors, inducing these offspring to be resistant to glucagon but sensitive to insulin (9).

Thus, although these results support the theory that LLP and LP offspring are insulin sensitive at a young age, an increase in insulin sensitivity alone does not explain the enhanced glycogen storage seen in the LLP and LP offspring. We did not find any changes in GLUT2 expression at either $10 \mathrm{~d}$ or $26 \mathrm{~d}$ as a result of maternal PR, in agreement with results found in 
another model of intrauterine growth restriction in which there was no effect on GLUT2 expression from birth until $21 \mathrm{~d}(16)$. In fetal hepatocytes glucose transporter 1 is the predominant glucose transporter when glucose is low, with GLUT2 contributing under high glucose conditions (17). The results suggest that there is no change in glucose uptake via GLUT2 as GLUT2 expression and glucose concentrations are not different among the groups, but uptake may be increased via another glucose transporter.

The absence of consistent changes in gene expression and activity of GS and GP $a$ also suggest that changes in glycogen are not just caused by changes in insulin sensitivity. Insulin is involved in the acute and chronic regulation of GS and GP. Regulation of GS and GP $a$ can be posttranscriptional, accounting for independent changes in mRNA and activity $(18,19)$. Studies in diabetic rats suggest that insulin may affect GS activity by affecting synthesis, degradation, or catalytic efficiency. Insulin has been shown to affect GP activity by stabilization of its mRNA (19). The absence of change in GS activity in the LLP and LP offspring suggests that the increase in glycogen storage is not because of an increase in glycogen synthesis induced by increased sensitivity to insulin. However, whereas GPa activity was reduced in the 26-d animals, it is unlikely that the reductions are sufficient to explain the increased glycogen storage by decreased glycogenolysis. Thus it appears that other factors may be acting with insulin to induce this enhanced glycogen storage.

Maternal undernutrition is reported to be associated with increased exposure of the fetus to glucocorticoids (20). Glucocorticoids are known stimulators of fetal hepatic glycogen deposition and inactivators of GP (21). Glucocorticoids are also known stimulators of leptin production (22). In this study, the LLP and LP offspring had greater leptin when adjusted for adiposity compared with the CONT offspring, as has been shown previously (23), whereas hyperleptinemia has been associated with reduced body weight in adult offspring (24). This result may be related to low insulin levels, resulting in reduced inhibitory feedback via the adipoinsular axis on the secretion of leptin by adipocytes (25). Another factor that has been shown to alter circulating leptin levels is dietary macronutrient composition; it has been shown that feeding of LP diets to dams results in altered milk macronutrient composition (26).

Leptin has been reported to have both insulin-like and anti-insulin-like effects on hepatic glucose metabolism, with these actions being dependent on both substrate or nutrient availability and on the prevailing insulinemia. In vivo, acute leptin treatment during hyperinsulinemic clamp studies in rats was shown to enhance the suppression of glycogenolysis by insulin (27). Hyperleptinemia induced by adenovirus therapy in normal rats resulted in a sparing of glycogen stores during the fed-to-fasted transition without any change in GP $a$ or GS activities (28). A single intraperitoneal injection of leptin reduced hepatic glycogen stores in gold thioglucose-obese mice, possibly by an indirect inhibitory effect on insulin secretion (11). In an in vitro study using rat hepatocytes, leptin had additive effects with insulin in inhibiting GPa activity while having no effect on glucokinase (GK) or GS activities (29). A recent paper suggests that leptin can act through some of the components of the insulin signaling cascade and so can modify (either enhance or inhibit) insulin-induced changes in gene expression (30). Therefore, leptin and insulin may be acting together to enhance glycogen storage in the LLP and LP offspring.

\section{CONCLUSIONS}

In conclusion, these studies show that maternal protein energy restriction results in reduced growth with reduced adipose tissue, liver weights, and insulin concentrations. Despite this, the offspring maintain equivalent total glycogen stores compared with CONT offspring. The exact mechanism for this has not been determined but may involve increased insulin sensitivity and glucose tolerance in the LLP and LP offspring in addition to an alternative non-insulin-dependent glycogen storage mechanism.

Acknowledgments. The authors thank Penfords Aust. P/L (formerly Starch Australasia) for their kind donation of maize starch and dextrose monohydrate.

\section{REFERENCES}

1. Hoet JJ, Hanson MA 1999 Intrauterine nutrition: its importance during critical periods for cardiovascular and endocrine development. J Physiol (Lond) 514:617-627

2. Holness MJ, Langdown ML, Sugden MC 2000 Early-life programming of susceptibility to dysregulation of glucose metabolism and the development of type 2 diabetes mellitus. Biochem J 349:657-665

3. Hales CN, Barker DJ 1992 Type 2 (non-insulin-dependent) diabetes mellitus: the thrifty phenotype hypothesis. Diabetologia 35:595-601

4. Ozanne SE 2001 Metabolic programming in animals. Br Med Bull 60:143-152

5. Hales CN, Desai M, Ozanne SE, Crowther NJ 1996 Fishing in the stream of diabetes: from measuring insulin to the control of fetal organogenesis. Biochem Soc Trans 24:341-350

6. Holness MJ, Sugden MC 1999 Antecedent protein restriction exacerbates development of impaired insulin action after high-fat feeding. Am J Physiol 276:E85-E93

7. Desai M, Byrne CD, Zhang J, Petry CJ, Lucas A, Hales CN 1997 Programming of hepatic insulin-sensitive enzymes in offspring of rat dams fed a protein-restricted diet. Am J Physiol 272:G1083-G1090

8. Ravelli AC, van der Meulen JH, Michels RP, Osmond C, Barker DJ, Hales CN, Bleker OP 1998 Glucose tolerance in adults after prenatal exposure to famine. Lancet 351:173-177

9. Ozanne SE, Smith GD, Tikerpae J, Hales CN 1996 Altered regulation of hepatic glucose output in the male offspring of protein-malnourished rat dams. Am J Physiol 270:E559-E564

10. Chan TM, Exton JH 1976 A rapid method for the determination of glycogen content and radioactivity in small quantities of tissue or isolated hepatocytes. Anal Biochem 71:96-105

11. Bryson JM, Phuyal JL, Proctor DR, Blair SC, Caterson ID, Cooney GJ 1999 Plasma insulin rise precedes rise in $o b$ mRNA expression and plasma leptin in gold thioglucose-obese mice. Am J Physiol 276:E358-E364

12. Wang MY, Koyama K, Shimabukuro M, Mangelsdorf D, Newgard CB, Unger RH 1998 Overexpression of leptin receptors in pancreatic islets of Zucker diabetic fatty rats restores GLUT-2, glucokinase, and glucose-stimulated insulin secretion. Proc Natl Acad Sci USA 95:11921-11926

13. Thomas JA, Schlender KK, Larner J 1968 A rapid filter paper assay for UDPglucoseglycogen glucosyltransferase, including an improved biosynthesis of UDP-14Cglucose. Anal Biochem 25:486-499

14. Gilboe DP, Larson KL, Nuttall FQ 1972 Radioactive method for the assay of glycogen phosphorylases. Anal Biochem 47:20-27

15. Snoeck A, Remacle C, Reusens B, Hoet JJ 1990 Effect of a low protein diet during pregnancy on the fetal rat endocrine pancreas. Biol Neonate 57:107-118

16. Lane RH, Crawford SE, Flozak AS, Simmons RA 1999 Localization and quantification of glucose transporters in liver of growth-retarded fetal and neonatal rats. Am J Physiol 276:E135-E142

17. Levitsky LL, Zheng Q, Mink K, Rhoads DB 1994 GLUT-1 and GLUT-2 mRNA, protein, and glucose transporter activity in cultured fetal and adult hepatocytes. Am J Physiol 267:E88-E94

18. Gannon MC, Nuttall FQ 1997 Effect of feeding, fasting, and diabetes on liver glycogen synthase activity, protein, and mRNA in rats. Diabetologia 40:758-763

19. Rao PV, Pugazhenthi S, Khandelwal RL 1995 The effects of streptozotocin-induced diabetes and insulin supplementation on expression of the glycogen phosphorylase gene in rat liver. J Biol Chem 270:24955-24960

20. Nyirenda MJ, Lindsay RS, Kenyon CJ, Burchell A, Seckl JR 1998 Glucocorticoid exposure in late gestation permanently programs rat hepatic phosphoenolpyruvate 
carboxykinase and glucocorticoid receptor expression and causes glucose intolerance in adult offspring. J Clin Invest 101:2174-2181

21. Stalmans W, Laloux M 1979 Glucocorticoids and hepatic glycogen metabolism Monogr Endocrinol 12:517-533

22. Bradley RL, Cheatham B 1999 Regulation of $o b$ gene expression and leptin secretion by insulin and dexamethasone in rat adipocytes. Diabetes 48:272-278

23. Rajakumar PA, He J, Simmons RA, Devaskar SU 1998 Effect of uteroplacental insufficiency upon brain neuropeptide $\mathrm{Y}$ and corticotropin-releasing factor gene expression and concentrations. Pediatr Res 44:168-174

24. Vickers MH, Breier BH, Cutfield WS, Hofman PL, Gluckman PD 2000 Fetal origins of hyperphagia, obesity, and hypertension and postnatal amplification by hypercaloric nutrition. Am J Physiol Endocrinol Metab 279:E83-E87

25. Kieffer TJ, Habener JF 2000 The adipoinsular axis: effects of leptin on pancreatic beta-cells. Am J Physiol Endocrinol Metab 278:E1-E14
26. Langley-Evans SC, Phillips GJ, Benediktsson R, Gardner DS, Edwards CR, Jackson AA, Seckl JR 1996 Protein intake in pregnancy, placental glucocorticoid metabolism and the programming of hypertension in the rat. Placenta 17:169-172

27. Rossetti L, Massillon D, Barzilai N, Vuguin P, Chen W, Hawkins M, Wu J, Wang J 1997 Short term effects of leptin on hepatic gluconeogenesis and in vivo insulin action. J Biol Chem 272:27758-27763

28. O’Doherty RM, Anderson PR, Zhao AZ, Bornfeldt KE, Newgard CB 1999 Sparing effect of leptin on liver glycogen stores in rats during the fed-to-fasted transition. Am J Physiol 277:E544-E550

29. Aiston S, Agius L 1999 Leptin enhances glycogen storage in hepatocytes by inhibition of phosphorylase and exerts an additive effect with insulin. Diabetes 48:15-20

30. Szanto I, Kahn CR 2000 Selective interaction between leptin and insulin signaling pathways in a hepatic cell line. Proc Natl Acad Sci USA 97:2355-2360 